\section{Des glycosidases bactériennes pour du sang universel}

Gerlind Sulzenbacher, Yves Bourne, Bernard Henrissat
Architecture et Fonction des Macromolécules Biologiques, UMR6098, CNRS, Universités Aix-Marseille I et II, Case 932, 163, avenue de Luminy, 13288 Marseille Cedex 9, France. Gerlind.Sulzenbacher@afmb.univ-mrs.fr.

Bernard.Henrissat@afmb.univ-mrs.fr
> Le système $A B O$, découvert par Karl Landsteiner en 1900 [1], détermine les règles principales qui régissent la transfusion sanguine. Ce système met en jeu des chaînes d'oligosaccharides qui portent un sucre terminal immunodéterminant, $\mathrm{N}$-acétyl-galactosamine dans le cas de l'antigène $A$ ou galactose dans le cas de l'antigène $B$, à la surface des érythrocytes (Figure 1). La surface des globules rouges du type $A B$ est recouverte des deux antigènes, tandis que les chaînes oligosaccharidiques qui caractérisent le groupe 0 sont dépourvues de sucre immunodéterminant. Lors d'une transfusion incompatible, les anticorps du receveur se fixent spécifiquement aux antigènes à la surface des globules rouges du donneur, provoquant ainsi l'agglutination et parfois l'hémolyse de ces derniers. C'est précisément en raison de l'absence de sucre antigénique que le groupe sanguin 0 est considéré universel et que les globules rouges 0 peuvent être transfusés à l'ensemble des individus des groupes $A, B$ et $A B^{l}$.

D'où l'idée visionnaire de Jack Goldstein, dans les années 1980, d'utiliser des enzymes pour enlever les sucres immuno-déterminants des antigènes $A$ et $B$ et engendrer ainsi des globules rouges du type 0 , soit du type donneur universel (appelés aussi cellules \&CO, enzyme-converted type 0) [2]. Goldstein a montré la faisabilité de l'approche en transformant des

Travail effectué en collaboration avec la Société ZymeQuest Inc., 100 Cummings Center, Suite 436H, Beverly MA, ÉtatsUnis.

${ }^{1}$ Les autres systèmes, tels que le facteur Rhésus, ne seront pas abordés dans cette étude. érythrocytes du type $B$ en cellules $B$ $\varepsilon C 0$ en utilisant une $\alpha$-galactosidase extraite de grains de café. Malheureusement le processus s'est avéré à la fois trop coûteux et peu efficace, car il fallait plus d'un gramme d'enzyme pour convertir une unité d'érythrocytes (200 ml de sang). En outre l'enzyme provenant des grains de café possède un maximum d'activité à $\mathrm{pH}$ acide, peu propice à la survie des globules rouges. Pour la transformation d'érythrocytes de type $A$ en $A-\varepsilon C O$, d'autres travaux ont été rapportés, utilisant plusieurs $\alpha-\mathrm{N}$-acétylgalactosaminidases, mais aucune ne s'est révélée véritablement efficace [3-5]. La raison de ces échecs tenait sans doute au fait que la recherche d'enzymes susceptibles d'éliminer le sucre immuno-déterminant avait été effectuée en utilisant des substrats du type para-nitro-phényl-galactose ou para-

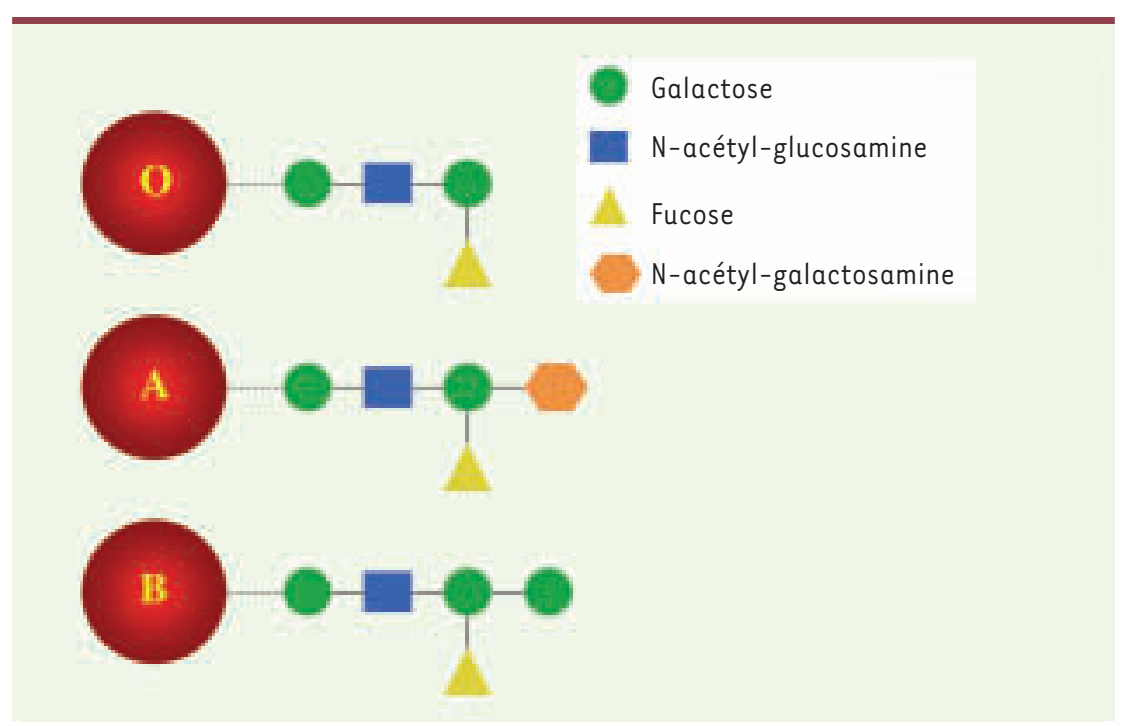

Figure 1. Antigènes du système ABO.
nitro-phényl-N-acétylgalactosamine. Ces substrats, couramment utilisés en enzymologie, ne ressemblent que très vaguement aux véritables molécules situées à la surface des érythrocytes et sont dépourvus en particulier de la chaîne complète d'oligosaccharides constituant les antigènes $A$ et $B$. La solution fut trouvée par nos collaborateurs, les chercheurs de la société ZymeQuest, qui ont utilisé des dérivés amino-coumarine des tétrasaccharides complets correspondant aux antigènes $A$ et $B$ pour cribler des milliers d'extraits cellulaires bactériens et fongiques. C'est ainsi qu'ils ont découvert des souches bactériennes produisant des $\alpha$ $\mathrm{N}$-acétylgalactosidases et des $\alpha$-galactosidases possédant une spécificité et une activité envers les antigènes du type $A$ et $B$ bien plus élevées que celles des enzymes connues jusqu'alors [6]. Ainsi pour transformer $200 \mathrm{ml}$ de sang, il suffit de 15-60 mg de $\alpha$-N-acétylgalactosidase 
pour le type A et $2 \mathrm{mg}$ de $\alpha$-galactosidase pour le type B. Les enzymes, qui peuvent êtres produites en grande quantité par expression recombinante dans des bactéries inoffensives, agissent à $\mathrm{pH}$ neutre et peuvent être facilement éliminées par simple dialyse une fois la conversion effectuée. Le traitement des cellules A$\varepsilon C O$ et B-દCO avec des anticorps hautement spécifiques pour les antigènes $A$ et $B$ ne révèle aucune présence résiduelle de ces derniers, indiquant que la transformation peut être considérée comme complète. L'ensemble de ces propriétés rend les deux enzymes extrêmement intéressantes pour la transformation des groupes sanguins $A, B$ et $A B$ en 0 .

La séquence en acides aminés des deux enzymes ne présente aucune similitude avec les quelques 30000 enzymes capables d'hydrolyser des sucres répertoriées dans la banque de données CAZy (http://www. cazy.org) créée et mise à jour dans notre laboratoire [7]. Dans cette base de données, les enzymes sont classées en familles tenant compte du mécanisme catalytique et de la structure tridimensionnelle. Cette

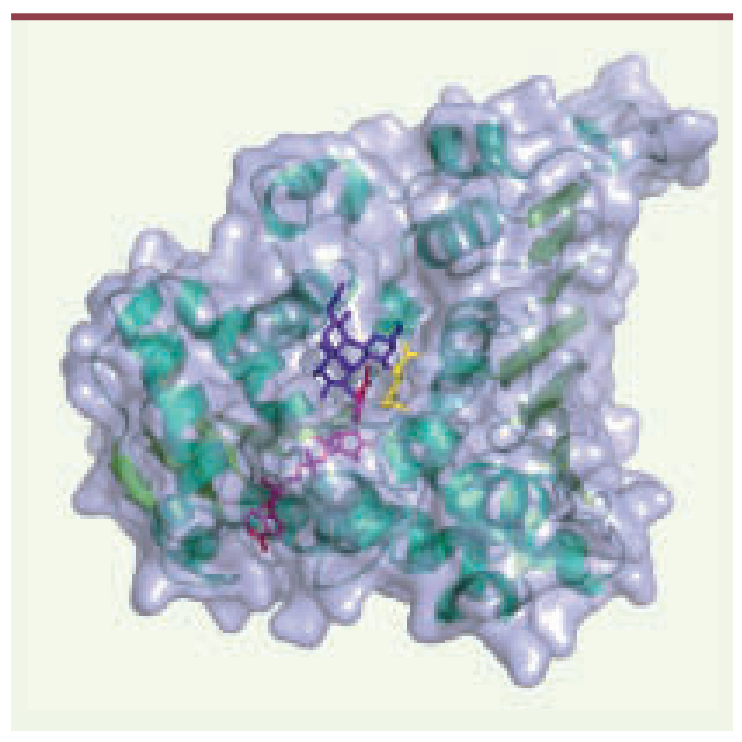

Figure 2. Vue générale de la structure moléculaire de l' $\alpha-N-$ acétylgalactosaminidase d'Elizabethkingia meningosepticum (vert) sous surface transparente (bleu clair) en complexe avec le cofacteur NAD+ (magenta) et l'antigène $A$ (violet), présent à la surface des globules rouges du type $A$. La molécule d' $\alpha$ $\mathrm{N}$-acétylgalactosamine, qui est reconnue et hydrolysée par l'enzyme, est colorée en jaune. classification permet d'établir des relations séquence-structure-fonction très précises. Les deux enzymes découvertes ont ainsi permis la définition de deux nouvelles familles de glycosidases, chacune regroupant exclusivement des enzymes d'origine bactérienne: la famille GH109 pour les $\alpha$-N-acétylgalactosidases et la famille GH110 pour les $\alpha$-galactosidases (pour une liste complète, consulter la base de données (AZy).

À notre grande surprise, l'analyse de la séquence en acides aminés de l' $\alpha-\mathrm{N}$ acétylgalactosaminidase de la bactérie Elizabethkingia meningosepticum, l'enzyme capable de cliver le sucre immunodéterminant de l'antigène $A$, a indiqué que celles-ci présentait des similitudes avec des oxydoréductases! Pour éliminer tous les doutes sur la fonction exacte de l'enzyme, la résolution de la structure tridimensionnelle par cristallographie aux rayons $X$ a été réalisée dans notre laboratoire. La technique permet de déterminer la position de chacun des dizaines de milliers d'atomes qui composent l'enzyme, et ainsi d'obtenir une image moléculaire dévoilant le mécanisme mis en oeuvre pour effectuer l'hydrolyse sélective observée. La structure tridimensionnelle que nous avons mise à jour (Figure 2) montre que l'enzyme possède une forte ressemblance structurale avec les oxydoréductases, en particulier par le repliement général de la chaîne polypeptidique, la présence du cofacteur nicotinamide-adénine-di-nucléotide, $N A D^{+}$, et l'organisation spatiale des acides aminés formant le centre actif. Celui-ci révèle une similitude frappante avec celui des glycosidases de la famille GH4 en dépit de repliements globalement différents des chaînes polypeptidiques. Depuis les premiers travaux sur le mécanisme catalytique des glycosidases dans les années 1950 [8], et après les nombreux progrès fondamentaux en enzymologie mécanistique réalisés ces quinze dernières années, on pensait avoir compris le mode d'action des glycosidases dans le moindre détail: l'hydrolyse enzymatique des sucres faisant intervenir deux variantes d'un mécanisme catalytique du type acide-base [9]. Les travaux sur les enzymes de la famille GH4 ont montré très récemment que l'hydrolyse de la liaison glycosidique peut être catalysée également par un autre mécanisme comportant une succession d'étapes d'oxydation-élimination-addition-réduction [10]. La structure tridimensionnelle de l' $\alpha$-N-acétylgalactosaminidase révèle qu'il s'agit du deuxième exemple connu de détournement de structure préexistante, en l'occurrence d'oxydoréductases, suivi d'une évolution vers une nouvelle fonction adaptée à des voies métaboliques différentes.

Le travail de recherche fondamentale n'est pas terminé. Bien que très performante, l'enzyme de conversion des antigènes $A$ n'a pas encore l'efficacité de celle assurant la conversion des antigènes B. Il existe plusieurs approches (ingénierie rationnelle, évolution moléculaire dirigée) qui peuvent à présent être mises en œuvre pour améliorer les paramètres cinétiques de l'enzyme. Par ailleurs, il nous reste à déterminer la structure tridimensionnelle de l' $\alpha$-galactosidase de Bacteroides fragilis, l'enzyme qui élimine le sucre immuno-déterminant de l'antigène $B$. Ces travaux sont nécessaires pour comprendre l'efficacité remarquable de cette enzyme. $\diamond$

Glycosidases for the production of universal blood

\section{RéFÉRENCES}

1. Landsteiner K. Agglutination phenomena of normal human blood. Wien Klin Wochenschr 2001 ; $113: 768-9$.

2. Goldstein J, Siviglia G, Hurst R, et al. Group B erythrocytes enzymatically converted to group 0 survive normally in $A, B$, and 0 individuals. Science $1982 ; 215: 168-70$. 
3. Zhu A, Monahan C, Wang ZK, et al. Characterization of recombinant $\alpha$-galactosidase for use in seroconversion from blood group $B$ to 0 of human erythrocytes. Arch Biochem Biophys 1996 ; 327 : 324-9.

4. Bakunina IY, Kuhlmann RA, Likhosherstov LM, et al. $\alpha-\mathrm{N}$-acetylgalactosaminidase from marine bacterium Arenibacter latericius KMM 426T removing blood type specificity of A-erythrocytes. Biochemistry (Mosc) 2002; 67:689-95.
5. Hsin-Yeh H, Chapman LF, Calcutt MJ, et al. Recombinant Clostridium perfringens $\alpha-\mathrm{N}$-acetylgalactosaminidase blood group A2 degrading activity. Artif Cells Blood Substit Immobil Biotechnol 2005 ; 33 : 187-99.

6. Liu $Q P$, Sulzenbacher $G$, Yuan $H$, et al. Bacterial glycosidases for the production of universal red blood cells. Nat Biotechnol 2007 ; 25 : 454-64.

7. Henrissat B, Bairoch $A$. Updating the sequence-based classification of glycosyl hydrolases. Biochem J 1996 ; $316: 695-6$.
NOUVELLE

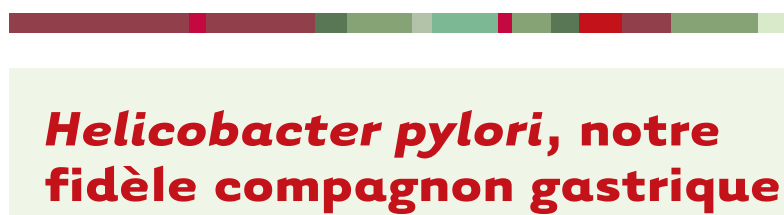

François Balloux

$>$ Ces dernières années ont vu l'émergence d'un quasi-consensus sur l'histoire de la colonisation du monde par l'homme anatomiquement moderne. Si de nombreux détails restent à clarifier, seule une minorité de chercheurs dans le domaine met en doute une origine africaine récente ( 200 000 ans). II y a 60000 ans environ, une expansion démographique a entraîné une sortie hors de l'Afrique et la colonisation de tous les continents Ce scénario est fondé sur l'analyse de marqueurs génétiques haploïdes (ADN mitochondrial et chromosome y) $[1,2]$, de marqueurs autosomiques [3-5] ainsi que de données morphologiques [6] et archéologiques [7].

\section{Dater l'association}

entre l'homme et ses pathogènes

Ces mouvements migratoires depuis une population ancestrale africaine expliquent pourquoi la variabilité génétique a une distribution géographique caractéristique. Les populations génétiquement les plus diverses se trouvent sur le continent africain. Le polymorphisme décroît de façon linéaire au fur et à mesure que I'on s'éloigne de l'Afrique, si cette distance est estimée comme la distance la plus courte possible en restant sur la terre ferme autant que possible $[3,5]$. Cela peut s'expliquer par la colonisation de proche en proche qui a entraîné, à chaque étape, une perte au hasard de certains des allèles présents dans la population parentale. Le phénomène peut être visualisé par analogie à la diffusion d'une goutte de colorant dans l'eau, qui, de façon transitoire, se traduira par une densité de pigments diminuant lorsque l'on s'éloigne du point d'impact de la goutte.

Ces progrès dans la description de la distribution géographique de la diversité génétique humaine ouvrent de nouvelles possibilités d'inférences sur l'âge de l'association entre notre espèce et les maladies infectieuses qui nous affectent. Schématiquement, plus l'association entre un pathogène et l'homme est ancienne, plus grande sera la similitude des distributions géographiques de leurs diversités génétiques. À l'exception de certaines maladies émergentes (SRAS ou syndrome respiratoire aigu sévère, VIH), on ne sait que très peu de choses sur l'âge des associations entre les humains et leurs pathogènes. Une hypothèse souvent avancée est que l'origine de la plupart des maladies infectieuses humaines est postérieure à la découverte de l'agriculture, il y a environ 10000 ans, dans la mesure où cette transition culturelle a entraîné des densités de population élevées.
8. Koshland DEJ. Stereochemistry and the mechanism of enzymatic reactions. Bio Rev $1953 ; 28: 416-36$.

9. Yip VL, Withers SG. Nature's many mechanisms for the degradation of oligosaccharides. Org Biomol Chem $2004 ; 2$ : 2707-13.

10. Yip VL, Varrot A, Davies GS, et al. An unusual mechanism of glycoside hydrolysis involving redox and elimination steps by a family $4 \alpha$-glycosidase from Thermotoga maritima. J Am Chem Soc 2004 ; $126: 8354-5$
Department of Genetics, University of Cambridge, Downing Street, Cambridge, CB2 $3 \varepsilon$ Royaume-Uni. fb255@gen.cam.ac.uk

\section{L'exemple d'Helicobacter pylori}

Un pathogène dont l'âge est particulièrement controversé est Helicobater pylori, une bactérie ne colonisant que l'estomac humain. Bien qu'infectant plus de la moitié de l'humanité, H. pylori n'a été décrite qu'en 1982 par deux chercheurs australiens, Robin Warren et Barry Marshall. D’emblée, Warren et Marshall étaient convaincus que $H$. pylori était impliquée dans la majorité des ulcères et des cancers de l'estomac. Ce point de vue n'étant pas partagé par la majorité de la communauté médicale, Marshall ingurgita une culture de ces bactéries, tomba fort malade puis se soigna avec des antibiotiques. Ce geste joua un rôle important dans la décision d'attribuer le prix Nobel de physiologie et de médecine 2005 aux deux chercheurs [8].

Il a été suggéré que $H$. pylori n'infecte I'homme que depuis très récemment [9]. Cette hypothèse a été remise en question lorsqu'il a été démontré que les populations sibériennes et amérindiennes étaient porteuses de souches apparentées, suggérant que les premiers hommes à s'être établi en Amérique après avoir traversé le Détroit de Béring il y a 10000 à 20000 ans étaient potentiellement déjà infectés [10]. 